Case Reports

\title{
A Reasonable Outcome with Systemic Corticosteroids in a Case of Ibuprofen Induced Stevens-Johnson Syndrome
}

\author{
${ }^{1,3}$ Elif Güdeloğlu and ${ }^{2}$ Volkan Emir Altan \\ ${ }^{1}$ Department of Pediatrics, Dr. Behçet Uz Children's Hospital of İzmir Turkey, Turkey \\ ${ }^{2}$ Department of Ophthalmology, Dr. Behçet Uz Children's Hospital of İzmir Turkey, Turkey \\ ${ }^{3}$ Department of Pediatrics, Turkey-Somalia Mogadishu Training and Research Hospital, Turkey
}

\author{
Article history \\ Received: 19-05-2020 \\ Revised: 07-08-2020 \\ Accepted: 04-09-2020 \\ Corresponding Author: \\ Elif Güdeloğlu \\ Department of Pediatrics, Dr. \\ Behçet Uz Children's Hospital \\ of İzmir Turkey, Turkey \\ Email: drelif55@hotmail.com
}

\begin{abstract}
Both Stevens-Johnson Syndrome (SJS) and Toxic Epidermal Necrolysis (TEN) are idiosyncratic severe mucocutaneous reactions, usually to drugs or infections, characterized by blistering and epithelial sloughing. Although rare, SJS and TEN are destructive diseases; in serious cases the acute phase may be followed by a variety of systemic complications, including multiorgan failure and death. Ibuprofen is a universal antipyretic and analgesic non-steroidal antiinflammatory drug which is widely used in practice and considered as relatively safe. Hereby we introduce an Ibuprofen induced SJS case in a 14-year-old Somalian female with chief complaint of extensive skin rashes accompanied by ulceration, mild fever and difficulty in swallowing for two days. Soon after the patient was diagnosed as Ibuprofen associated SJS, she was treated with systemic corticosteroids in addition to general measures. The sign and symptoms started to resolute as soon as the second day of approval and at the seventh day the patient was discharged from the hospital. It was noteworthy that the findings of SJS/TEN appeared in relatively shorter time after the usage of accused drug compared to the previous knowledge, as well as it was remarkable. In addition, it is noticeable, that easily accessible drugs such as ibuprofen, which are frequently used in children, are not as innocent as it is thought. Moreover that is important to inform and closely follow the patients for the development of delayed drug hypersensitivity reactions.
\end{abstract}

Keywords: Ibuprofen, Stevens Johnson Syndrome, Systemic Steroid Treatment

\section{Introduction}

Ibuprofen is a universal antipyretic and analgesic non-steroidal antiinflammatory drug which inhibits cyclooxygenase (prostaglandin-synthase) enzyme. Moreover, it tarnishes the final transformation of arachidonic acid to prostaglandins, prostacyclin and thromboxanes. Ibuprofen induced hypersensitivitysyndrome is a host dependent idiosyncratic drug-reaction (Nanau and Neuman, 2010). The drug-induced Type IV hypersensitivity reaction is thought to play a role in the development of SJS and TEN; its pathogenesis has not been fully elucidated (Belver et al., 2016; FerrandizPulido and Garcia-Patos, 2013). The disease most likely occurs as a result of the binding of drugs or their metabolites to the surface of keratinocytes by becoming haptic and becoming antigenic (Roujeau, 1993).

Stevens Johnson Syndrome (SJS) and Toxic Epidermal Necrolysis (TEN) are rare mucocutaneous conditions, which are usually induced by drugs and being associated with a lengthened course, finalized with probable lethal conclusion. Both are noted as being two variants of the same course that they differ regarding the degree of the affected surface area (Schwartz et al., 2013). The major cause of death is mostly sepsis (Harr and French, 2010). The current management of SJS/TEN includes sudden withdrawal of the offending drug, stabilization with general measures like fluid replacement and adequate nutrition, assurance of analgesia, as well as maintenance of skin, mouth, eye and urogenital care, in addition to systemic immunomodulatory therapy (McPherson et al., 2019). The first symptoms of Stevens Johnson syndrome and TEN usually start 4-14 days after the drug intake begins, this period sometimes extend up to 3-6 weeks (Mawson et al., 2015).

Herein, we report an SJS case associated with ibuprofen usage which was successfully managed with 
systemic corticosteroids as main treatment. Written informed consent was obtained from the patient's family for publication of this case report.

\section{Case}

A 14-year-old Somalian female with a prior history of no known chronic disease or surgery, admitted to the Pediatric Emergency Department of a teachinghospital in central Mogadishu with chief complaint of extensive skin rashes with ulceration and difficulty in swallowing for two days. Two days before the appearance of skin rashes the child had complained upper airway infection presented with symptoms of high-grade fever, frequent vomiting and severe malaise. Afterwards, she had taken Ibuprofen 400-mg tablets 12 hourly for two days. Therefore, she developed multiple maculae initially over the trunk, then chest, face, lower limbs on the third day.
She was admitted to the Emergency and upon examination, had extensive erythema, necrosis and exfoliating bullous detachment of the epidermis and mucous membranes (conjunctival, oral and genital) (Fig. 1a to $1 \mathrm{~d}$ ). The body temperature was $38.3^{\circ} \mathrm{C}$, respiratory rate being 27 breaths/min, pulse rate 132 beats/min, blood pressure $110 / 75 \mathrm{~mm} \mathrm{Hg}$ and oxygen saturation 99\%. The acute case, typical clinical symptoms, rapid course, skin sensitivity, positive Nikolsky sign and skin biopsy performed in the patient's history were guiding in terms of SJS. The patient's failure to use any additional medication allowed us to easily understand that the agent responsible for the clinic was Ibuprofen. Therefore, the patient was diagnosed as Ibuprofen associated Stevens Johnson syndrome.

Firstly, Ibuprofen was immediately withdrawn and to maintain fluid-electrolyte balance the patient was supported with balanced electrolyte solution. In addition, a nasogastric tube inserted to maintain enteric nutrition.

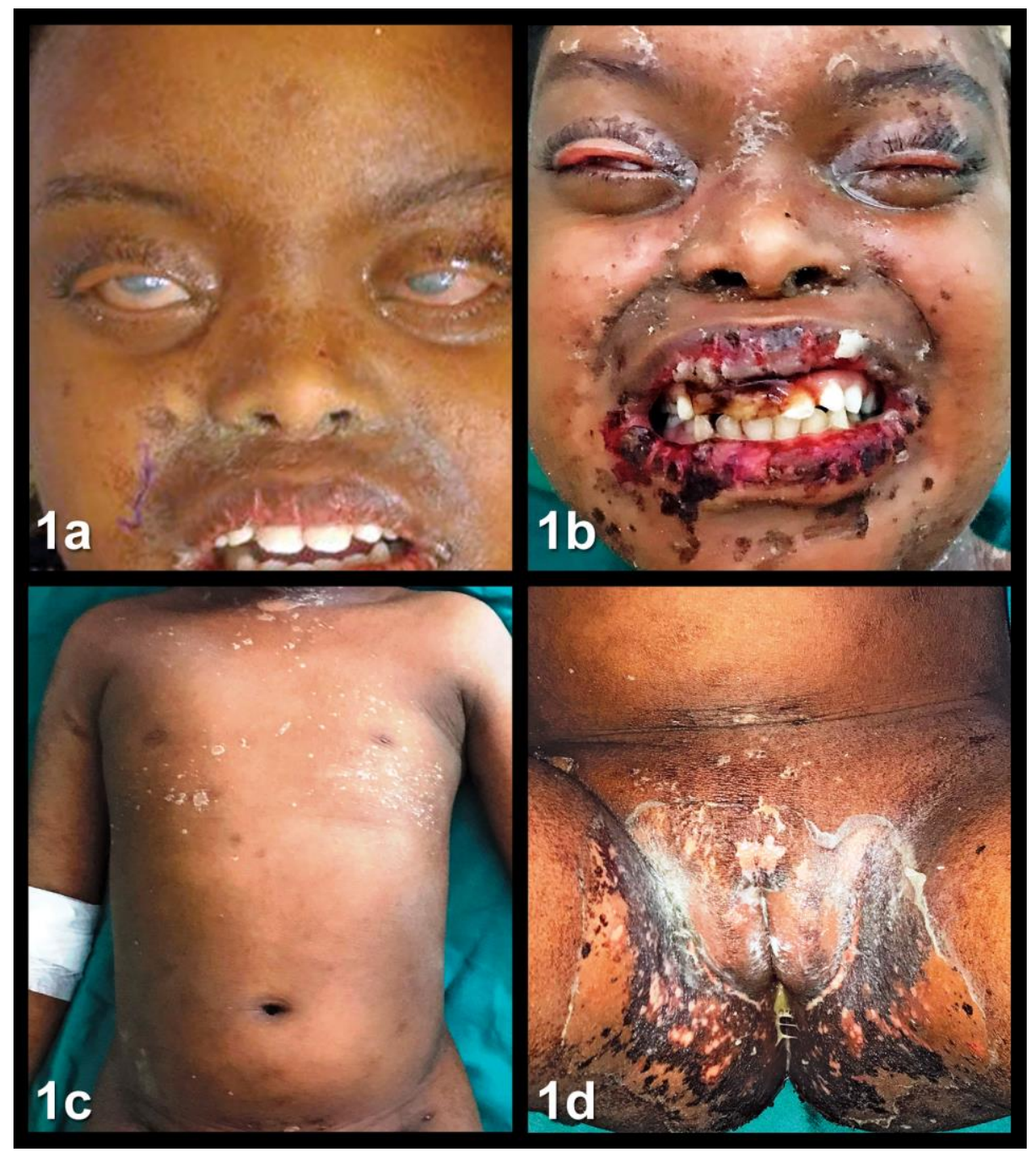

Fig. 1: (a) Eye findings of the patient taken as soon as possible; (b) Mouth findings at the approval; (c)Body and dermatologic findings at the approval; (d) Genital findings at the approval 


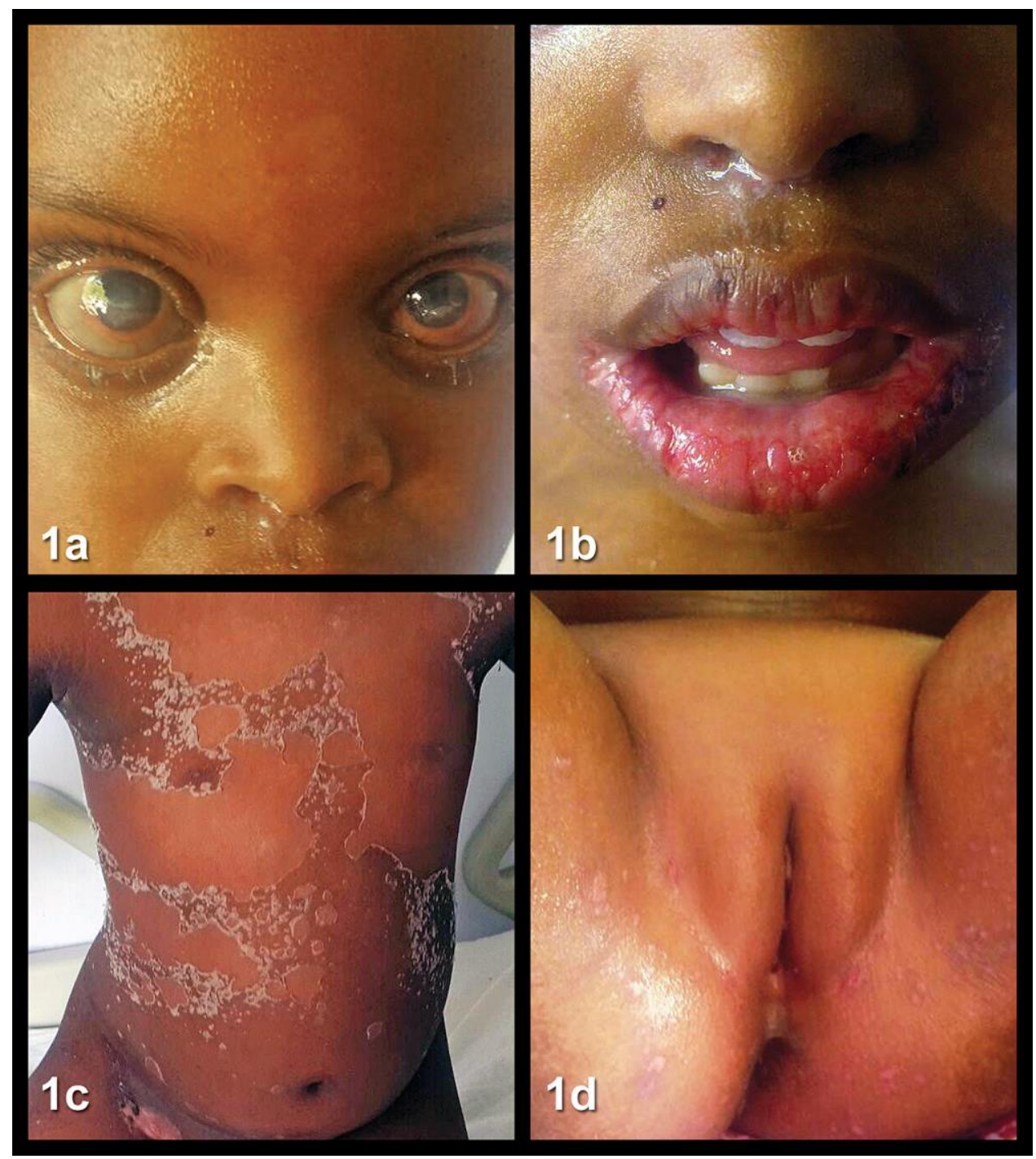

Fig. 2: (a) Eye findings of the patient at disposal; (b) Mouth findings at disposal; (c) Body and dermatologic findings at disposal; (d) Genital findings at disposal

She had also severe local ophthalmic complaints composed of severe conjunctival chemosis and keratopathy. Artificial tear driblets besides antibiotic eye drops and ointments were administered to both eyes to keep them moistened while preventing possible local infections. The patient had painful oral erosions with severe crusting of the lips and increased salivation. For oral lesions Miconazole oral gel was prescribed twice a day. Genitoanal region lesions treated by fusidic acid in combination with scatrizing and epithelizing creams.

Through the first five days of admission high dose of intravenous methylprednisolone ( $900 \mathrm{mg} /$ day) has been given. The patient was not given any systemic antibiotic therapy. Even though in management of SJS, IVIg, ciclosporin, thalidomide, cyclophosphamide, TNF inhibitors, granulocyte-colony stimulating factor, plasmapheresis and haemoperfusion, hemodialysis, plasmapheresis and hyperbaric oxygen treatment are being suggested, unfortunately systemic steroids were only available options in Somalia at that time.

Systemic symptoms of sepsis has been dropped back on second day of admission. Skin and mucosal lesions started to faint as soon as the fifth day of the management (Fig. 2a to 2d). On the seventh day of treatment, the patient was discharged and followed out patiently.

\section{Discussion}

SJS is generally characterized by epidermal destruction in discrete locations all over the body in which the emanation usually abrupt. Roughly after several days of malaise, high body temperature and 
upper respiratory tract findings, the skin lesions take place, which makes the situation harder to be distinguished (Creamer et al., 2016). In our case dermatological findings erupted two days after the patient had begun to take Ibuprofen medication.

According to recent guides SJS/TEN represent a spectrum of reactive disorders with mucocuteneous involvement in which SJS, overlap SJS/TEN, TEN and respiratory infection induced rash and mucositis take place (McPherson et al., 2019). The involvement of epithelial detachment in our case was less than $10 \%$ of body surface area so that it was compatible with SJS.

Supportive measures have the utmost importance in the management of SJS/TEN. This includes care of skin, mucous membranes (ocular, urogenital and oral), resuscitation, fluid balance, nutritional support, analgesia, preventing life-threatening complications and long-term morbidity (Zimmermann et al., 2017; Struck et al., 2010).

Randomized controlled trial datum on the effectiveness of immunomodulatory regimens are currently insufficient, including systemic corticosteroids, IVIg, ciclosporin, thalidomide, cyclophosphamide, TNF inhibitors, granulocyte-colony stimulating factor, plasmapheresis and haemoperfusion. Despite lack of evidence, both systemic corticosteroids and IVIg are frequently used treatments regardless of patients age. In addition, due to shortage of supplies at that time in Somalia, the only available option was usage systemic steroids (McPherson et al., 2019).

Nevertheless, our case needed no more investigation and management modalities, resolving in following days of treatment. Finally, she was discharged in a good and functional condition from the hospital as an outpatient.

\section{Conclusion}

Although today intravenous immunoglobulins, immunomodulators, hemodialysis, plasmapheresis and hyperbaric oxygen are being started to be addressed in management of SJS; due to the regional restrictions of drug availability, our only option was being high dose steroid treatment. This case shows that in spite of novel treatment options for SJS, management with systemic corticosteroids is probably an effective choice and should not be underrated.

\section{Authors Contributions}

All authors attest that they meet the current ICMJE criteria for Authorship

\section{Ethics}

This article is original and contains unpublished material. The corresponding author confirms that all of the other authors have read and approved the manuscript and no ethical issues involved.

\section{References}

Belver, M. T., Michavila, A., Bobolea, I., Feito, M., Bellón, T., \& Quirce, S. (2016). Severe delayed skin reactions related to drugs in the paediatric age group: a review of the subject by way of three cases (Stevens-Johnson syndrome, toxic epidermal necrolysis and DRESS). Allergologia et immunopathologia, 44(1), 83-95.

Creamer, D., Walsh, S. A., \& Dziewulski, P. (2016). U.K. guidelines for the management of StevensJohnson syndrome/toxic epidermal necrolysis in adults 2016. Br J Dermatol. 174, 1194-227.

Ferrandiz-Pulido, C., \& Garcia-Patos, V. (2013). A review of causes of Stevens-Johnson syndrome and toxic epidermal necrolysis in children. Archives of disease in childhood, 98(12), 998-1003.

Harr, T., \& French, L. E. (2010). Toxic epidermal necrolysis and Stevens-Johnson syndrome. Orphanet journal of rare diseases, 5(1), 39.

Mawson, A. R., Eriator, I., \& Karre, S. (2015). Stevens-Johnson syndrome and toxic epidermal necrolysis (SJS/TEN): could retinoids play a causative role?. Medical Science Monitor: International Medical Journal of Experimental and Clinical Research, 21, 133.

McPherson, T., Exton, L. S., Biswas, S., Creamer, D., Dziewulski, P., Newell, L., ... \& Walker, S. (2019). British Association of Dermatologists' guidelines for the management of Stevens-Johnson syndrome/toxic epidermal necrolysis in children and young people, 2018. British Journal of Dermatology, 181(1), 37-54.

Nanau, R. M., \& Neuman, M. G. (2010). Ibuprofeninduced hypersensitivity syndrome. Translational Research, 155(6), 275-293.

Roujeau, J. C. (1993). Drug-induced toxic epidermal necrolysis. II. Current aspects. Clinics in dermatology, 11(4), 493-500.

Schwartz, R. A., McDonough, P. H., \& Lee, B. W. (2013). Toxic epidermal necrolysis: Part I. Introduction, history, classification, clinical features, systemic manifestations, etiology and immunopathogenesis. Journal of the American Academy of Dermatology, 69(2), 173-e1.

Struck, M. F., Illert, T., Liss, Y., Bosbach, I. D., Reichelt, B., \& Steen, M. (2010). Toxic epidermal necrolysis in pregnancy: case report and review of the literature. Journal of burn care \& research, 31(5), 816-821.

Zimmermann, S., Sekula, P., Venhoff, M., Motschall, E., Knaus, J., Schumacher, M., \& Mockenhaupt, M. (2017). Systemic immunomodulating therapies for Stevens-Johnson syndrome and toxic epidermal necrolysis: a systematic review and meta-analysis. JAMA dermatology, 153(6), 514-522. 